\title{
Analysis of Exponential Logarithmic Lifetime Distribution
}

\author{
Anoop Chaturvedi ${ }^{1 *}$ and Maneesh Kumar Dubey ${ }^{2}$ \\ ${ }^{1}$ Department of Statistics, University of Allahabad, Allahabad, India \\ ${ }^{2 *}$ Department of Statistics, University of Allahabad, Allahabad, India \\ *Corresponding Author: maneesh231001@gmail.com
}

Available online at: www.isroset.org

Received 20/Jan/2018, Revised 28/Jan/2018, Accepted 22/Feb/2018, Online 28/Feb/2018

\begin{abstract}
This paper describes the Bayesian inference and prediction of the Exponential Logarithmic distribution . The aim of this paper is to obtain the Bayesian inference of the unknown parameters under different loss functions. The Bayes estimates can be obtained and it has been used to compute the Bayes estimates and also to construct symmetric loss function. We consider the posterior predictive density of the future observations and also asymmetric loss function.
\end{abstract}

Keywords- Lifetime distributions, reliability, failure rate, order statistics, exponential distribution, truncated logarithmic distribution

\section{INTRODUCTION}

Exponential distributions are encountered as lifetime distribution with constant rate. They are commonly employed in the formation of models of lifetime distribution, stochastic process in general. The distributions with decreasing failure rate are studied in the works of Lomax (1954) Porchan (1963), Barlow et al (1963), Borlow and Marshall (1964,1965), Marshall and Porshan (1965), Cozzolino(1968),Dahiya and Garland (1972), Mcnolty et al (1980), Saunders and Myhre (1983), Nassar (1988), Gleser (1989) , Gurland and Sethurman (1994), admids and Loukas (19980 and Kus (2007).

It is remarkable that the development of appropriate Bayesian inference procedures has been quite limited. Zelllener (1997) includes an example involving Bayesian inference in the classical Pareto distribution for the special case in which scale parameter is known .Malik (1970) and earlier, Muniruzzaman (1968) were apparently the first to consider the case known scale parameter. Sinhaand Howlader (1980) studied Bayesian estimators in the known scale case .Arnold and Press (1983, 1986) discussed possible forms of priors and Bayesian analysis for different cases Pandey et al (1986)and Bhattacharya et al (1992) provides a recent through study of Bayesian estimation on the subject.

Despite being a Gamma family of distributions discussed by Karl person as early as 1895 , it took another three and half decades for exponential distribution to appear in its own in the statistical literature .Kondo (1931) referred to the exponential distribution of standard deviation, as Pearson 's $\mathrm{X}$ type $\mathrm{X}$ distribution.

In the context of survival analysis Basu and Ebrahimi $(1988,1991)$, Chaturvedi (1998) used this loss function for estimating the mean life time and survival analysis of the exponential model for different priors. Rojo (1987),Rai (1996),Pandey (1997) have applied the Linex loss function in various other estimation problems.

The exponential logarithmic (EL) lifetime distribution has been developed by mixing exponential distribution with logarithmic distribution and has decreasing failure rate (DFR). The present chapter considers the Bayesian analysis of EL distribution under appropriate prior distributions and derives the posterior distributions from the parameters. The Bayes estimators for different parameters and survival function have been derived under squared error loss function and LINEX loss function. In this chapter also we derived the posterior analysis of exponential logarithmic distribution under type 1 censoring and derive the survival function under squared error loss function and Linex loss function.

\section{EXPONENTIAL LOGARITHMIC LIFETIME MODEL}

Let us consider the exponential distribution with following probability distribution function (pdf)

$f_{y}(y ; \beta)=\beta e^{-\beta y} I_{(0, \infty)}^{y} ; \beta>0$ 
Further, let $\mathrm{Z}$ be a random variable following a logarithmic distribution with probability mass function

$P_{Z}(z ; p)=\frac{(1-p)^{Z}}{-Z \ln p} \mathrm{I}_{\{1,2,3, \ldots .\}}^{(\mathrm{y})}$

where $0<\mathrm{p}<1$ and $I_{A}^{(y)}$ is the indicator function defined as

$$
I_{A}^{(y)}= \begin{cases}1 & \text { ify } \in A \\ 0 & \text { ify } \notin A\end{cases}
$$

We assume that the random variables $\mathrm{Y}$ and $\mathrm{Z}$ are independent, Let us define, $\mathrm{X}=\min \left\{Y_{1}, Y_{2}, \ldots . Y_{n}\right\}$.Then the conditional pdf of $\mathrm{X}$ given $\mathrm{Z}$ is

$f_{x \mid z}(X \mid Z ; \beta)=\beta Z e^{-\beta x z}$

Further, the marginal pdf of $\mathrm{X}$ is

$f_{X}(x ; p, \beta)=\frac{-\beta(1-p) e^{-\beta x}}{-\ln p\left[1-(1-p) e^{-\beta x}\right]} I_{(0, \infty)}^{(x)}$

The above distribution $\mathrm{X}$ is referred as the exponential logarithmic (EL) distribution.

The probability distribution function of EL distribution is monotonic decreasing with modal value $\beta(1-p)(-p \ln p)$ at $\mathrm{x}=0$ for all values of parameters. The EL leads to exponential distribution with parameter $\beta$, as $p \rightarrow 1$.

The cumulative distribution function (cdf) of EL distribution is given by

$F_{X}(x, p, \beta)=1-\frac{\ln \left[1-(1-p) e^{-\beta x}\right]}{\ln p}$

Let $\mathrm{x}_{1}, \ldots, \mathrm{x}_{\mathrm{n}}$ be random variables from the EL distribution.

We can write the pdf of $X$ as

$$
\begin{aligned}
f_{X}(x, p, \beta) & =\frac{-\beta(1-p) e^{-\beta x}}{-\ln p\left[1-(1-p) e^{-\beta x}\right]} I_{(0, \infty)}^{(x)} \\
& =\frac{(1-p) \beta e^{-\beta x} \sum_{z=0}^{\infty}(1-p) e^{-\beta x z}}{(-\ln p)}
\end{aligned}
$$

$$
=\frac{\beta \sum_{z=0}^{\infty}(1-p)^{z+1} e^{-(z+1) \beta x}}{(-\ln p)^{n}}
$$

Hence the likelihood function is given by

$$
\begin{aligned}
& \prod_{i=1}^{n} f\left(x_{i} ; p, \beta\right) \\
& =\frac{\beta^{n}}{(-\ln p)^{n}} \prod_{i=1}^{n}\left\{\sum_{z=1}^{\infty}(1-p)^{z} e^{-\beta z x_{i}}\right\}
\end{aligned}
$$

$=\frac{\beta^{n} e^{-\frac{\beta}{\bar{\beta}}} \sum_{z_{1=1}}^{\infty} \sum_{z_{2=1}}^{\infty} \cdots \sum_{z_{n=1}}^{\infty}(1-p)^{\sum_{i=1}^{n} z_{i}} e^{-\beta \sum_{i=1}^{n} z_{i} x_{i}}}{(-\ln p)^{n}}$

\section{Posterior Analysis}

\section{Prior Distribution:}

Let us assume that for the purpose of Bayesian analysis exponential logarithmic distribution follows Gamma distribution as a prior distribution

$p(\beta \mid \bar{\beta}, \alpha) \propto \beta^{\alpha-1} e^{-\frac{\beta}{\bar{\beta}}}$

\section{Theorem 1}

The posterior distribution of $\beta$, under the known prior distribution given in equation (2.7) is defined as

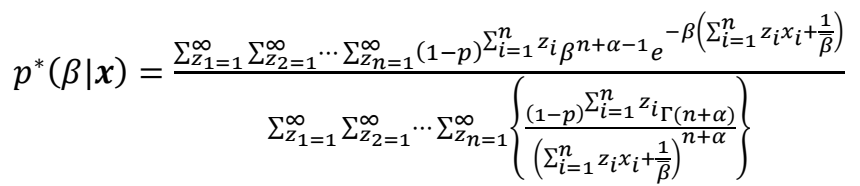

\section{Proof:}

We assume the prior distribution of $\beta$ as gamma distribution with pdf given by

$p(\beta \mid \bar{\beta}, \alpha) \propto \beta^{\alpha-1} e^{-\bar{\beta}}$

We assume that $\mathrm{p}$ is known. Further we write $\boldsymbol{x}=$ $\left(x_{1}, \ldots, x_{n}\right)$. 
Combining the likelihood function (4.2.6) with the prior distribution (3.2) we obtain the posterior distribution of $\beta$ as

$p^{*}(\beta \mid \boldsymbol{x})=\frac{\prod_{i=1}^{n} f\left(x_{i} ; p, \beta\right) p(\beta \mid \bar{\beta}, \alpha)}{p^{*}(\boldsymbol{x})}$

$=\frac{(-\ln p)^{-n} \sum_{z_{1=1}}^{\infty} \sum_{z_{2=1}}^{\infty} \cdots \sum_{z_{n=1}}^{\infty}(1-p)^{\sum_{i=1}^{n} z_{i}} \beta^{n+\alpha-1} e^{-\beta\left(\sum_{i=1}^{n} z_{i} x_{i}+\frac{1}{\beta}\right)}}{p^{*}(\boldsymbol{x})}$

where,

$$
\begin{aligned}
p^{*}(\boldsymbol{x})=\int_{0}^{\infty} \prod_{i=1}^{n} f\left(x_{i} ; p, \beta\right) p(\beta \mid \bar{\beta}, \alpha) d \beta \\
=(-\ln p)^{-n} \sum_{z_{1}=1}^{\infty} \sum_{z_{2}=1}^{\infty} \ldots \sum_{z_{n=1}}^{\infty}(1-p)^{\sum_{i=1}^{n} z_{i}} \\
\quad \times \int_{0}^{\infty} \beta^{n+\alpha-1} e^{-\beta\left(\sum_{i=1}^{n} z_{i} x_{i}+\frac{1}{\beta}\right)} d \beta \\
=(-\ln p)^{-n} \sum_{z_{1}=1}^{\infty} \sum_{z_{2}=1}^{\infty} \ldots \sum_{z_{n}=1}^{\infty}\left\{\frac{(1-p)^{\sum_{i=1}^{n} z_{i}} \Gamma(n+\alpha)}{\left(\sum_{i=1}^{n} z_{i} x_{i}+\frac{1}{\bar{\beta}}\right)^{n+\alpha}}\right\}
\end{aligned}
$$

Substituting the value of $p^{*}(\boldsymbol{x})$ in (3.3), the expression for the posterior distribution becomes

$p^{*}(\beta \mid \boldsymbol{x})$

$$
=\frac{\sum_{z_{1=1}}^{\infty} \sum_{z_{2=1}}^{\infty} \cdots \sum_{z_{n=1}}^{\infty}(1-p)^{\sum_{i=1}^{n} z_{i}} \beta^{n+\alpha-1} e^{-\beta\left(\sum_{i=1}^{n} z_{i} x_{i}+\frac{1}{\bar{\beta}}\right)}}{\sum_{z_{1=1}}^{\infty} \sum_{z_{2}=1}^{\infty} \cdots \sum_{z_{n=1}}^{\infty}\left\{\frac{(1-p)^{\sum_{i=1}^{n} z_{i}} \Gamma(n+\alpha)}{\left(\sum_{i=1}^{n} z_{i} x_{i}+\frac{1}{\bar{\beta}}\right)^{n+\alpha}}\right\}}
$$

\section{Estimation of Parameters ANd Survival}

\section{FUNCTION UNDER SQUARED ERROR LOSS}

For an estimator $\hat{\beta}$ of $\beta$ the squared error loss function (SELF) is defined as

$$
L_{1}=(\hat{\beta}-\beta)^{2}
$$

Under the above squared error loss function, the Bayes estimator of $\beta$ is the posterior mean given by

$\tilde{\beta}=E(\beta \mid x)$

\section{Theorem2}

The posterior mean of parameter $\beta$ of exponential Logarithmic distributions is defined under the prior distribution as given in equation (2.7) as

$$
\begin{aligned}
& \tilde{\beta}=(n+\alpha) \frac{\sum_{z_{1=1}}^{\infty} \sum_{z_{2}=1}^{\infty} \cdots \sum_{z_{n=1}}^{\infty}\left\{\frac{(1-p)^{\sum_{i=1}^{n} z_{i}}}{\left(\sum_{i=1}^{n} z_{i} x_{i}+\frac{1}{\bar{\beta}}\right)^{n+\alpha+1}}\right\}}{\sum_{z_{1=1}}^{\infty} \sum_{z_{2}=1}^{\infty} \cdots \sum_{z_{n=1}}^{\infty}\left\{\frac{(1-p)^{\sum_{i=1}^{n} z_{i}}}{\left(\sum_{i=1}^{n} z_{i} x_{i}+\frac{1}{\bar{\beta}}\right)^{n+\alpha}}\right\}} \\
& \text { Proof : }
\end{aligned}
$$

The posterior mean of $\beta$ is defined as

$$
\begin{aligned}
\delta^{*}(\boldsymbol{x}) & =E(\beta / \boldsymbol{x}) \\
& =\int_{0}^{\infty} \beta p^{*}(\beta \mid \boldsymbol{x}) d \beta
\end{aligned}
$$

where,

$p^{*}(\beta \mid \boldsymbol{x})=\frac{\prod_{i=1}^{n} f\left(x_{i} ; p, \beta\right) p(\beta \mid \bar{\beta}, \alpha)}{p^{*}(\boldsymbol{x})}$

Since the value of $p^{*}(\beta \mid x)$ is find from equation (3.5)

$$
p^{*}(\beta \mid \boldsymbol{x})=\frac{\sum_{z_{1}=1}^{\infty} \sum_{z_{2}=1}^{\infty} \cdots \sum_{z_{n=1}}^{\infty}(1-p)^{\sum_{i=1}^{n} z_{i} \beta^{n+\alpha-1} e^{-\beta\left(\sum_{i=1}^{n} z_{i} x_{i}+\frac{1}{\beta}\right)}}}{\sum_{z_{1}=1}^{\infty} \sum_{z_{2}=1}^{\infty} \cdots \sum_{z_{n}=1}^{\infty}\left\{\frac{(1-p)^{\sum_{i=1}^{n} z_{i}(n+\alpha)}}{\left(\sum_{i=1}^{n} z_{i} x_{i}+\frac{1}{\beta}\right)^{n+\alpha}}\right\}}
$$

Put the value of $p^{*}(\beta \mid x)$ from equation (3.5) in equation (4.4) we get 


$$
\begin{gathered}
=\int_{0}^{\infty} \beta \frac{\sum_{z_{1=1}}^{\infty} \sum_{z_{2=1}}^{\infty} \cdots \sum_{z_{n=1}}^{\infty}(1-p)^{\sum_{i=1}^{n} z_{i}} \beta^{n+\alpha-1} e^{-\beta\left(\sum_{i=1}^{n} z_{i} x_{i}+\frac{1}{\bar{\beta}}\right)}}{\sum_{z_{1=1}}^{\infty} \sum_{z_{2}=1}^{\infty} \cdots \sum_{z_{n=1}}^{\infty}\left\{\frac{(1-p)^{\sum_{i=1}^{n} z_{i} \Gamma(n+\alpha)}}{\left(\sum_{i=1}^{n} z_{i} x_{i}+\frac{1}{\bar{\beta}}\right)^{n+\alpha}}\right\}} d \beta \\
=(n+\alpha) \frac{\sum_{z_{1=1}}^{\infty} \sum_{z_{2}=1}^{\infty} \cdots \sum_{z_{n=1}}^{\infty}\left\{\frac{(1-p)^{\sum_{i=1}^{n} z_{i}}}{\left(\sum_{i=1}^{n} z_{i} x_{i}+\frac{1}{\bar{\beta}}\right)^{n+\alpha+1}}\right\}}{\sum_{z_{1=1}}^{\infty} \sum_{z_{2}=1}^{\infty} \cdots \sum_{z_{n=1}}^{\infty}\left\{\frac{(1-p)^{\sum_{i=1}^{n} z_{i}}}{\left(\sum_{i=1}^{n} z_{i} x_{i}+\frac{1}{\bar{\beta}}\right)^{n+\alpha}}\right\}}
\end{gathered}
$$

which is the required result

\section{Survival Function:}

Under the SELF the survival function for EL distribution is given by

$$
\begin{aligned}
S(t) & =P(X>t) \\
& =1-\left[1-\frac{\ln \left(1-(1-p) e^{-\beta t}\right)}{\ln p}\right] \\
& =\frac{\ln \left[1-(1-p) e^{-\beta t}\right]}{\ln p} \\
& =\frac{1}{(-\ln p)} \sum_{j=1}^{\infty} \frac{(1-p)^{j}}{j} e^{-j \beta t}
\end{aligned}
$$

Hence, under squared error loss function, the Bayes estimator of survival function is obtained as

$\tilde{S}(t)=\int_{0}^{\infty} S(t) p^{*}(\beta \mid \boldsymbol{x}) d \beta$

Put the value of $S(t)$ and $p^{*}(\beta \mid x)$ from equation (4.6) and equation (3.5) in equation (4.7) we get

$$
\begin{aligned}
& =\int_{0}^{\infty} \frac{1}{(-\ln p)} \sum_{j=1}^{\infty} \frac{(1-p)^{j}}{j} e^{-j \beta t} \\
& \times \frac{\sum_{z_{1=1}}^{\infty} \sum_{z_{2=1}}^{\infty} \cdots \sum_{z_{n=1}}^{\infty}(1-p)^{\sum_{i=1}^{n} z_{i}} \beta^{n+\alpha-1} e^{-\beta\left(\sum_{i=1}^{n} z_{i} x_{i}+\frac{1}{\bar{\beta}}\right)}}{\sum_{z_{1}=1}^{\infty} \sum_{z_{2=1}}^{\infty} \cdots \sum_{z_{n=1}}^{\infty}\left\{\frac{(1-p)^{\sum_{i=1}^{n} z_{i} \Gamma(n+\alpha)}}{\left(\sum_{i=1}^{n} z_{i} x_{i}+\frac{1}{\bar{\beta}}\right)^{n+\alpha}}\right\}} \\
& =\sum_{j=1}^{\infty} \sum_{z_{1}=1}^{\infty} \sum_{z_{2}=1}^{\infty} \cdots \sum_{z_{n=1}}^{\infty} \frac{(1-p)^{j}}{j}(1-p)^{\sum_{i=1}^{\infty} z_{i}} \times \\
& \frac{\int_{0}^{\infty} \beta^{n+\alpha-1} e^{-\beta\left(\sum_{i=1}^{n} z_{i} x_{i}+\frac{1}{\bar{\beta}}+j t\right)} d \beta}{(-\ln p) \sum_{z_{1=1}}^{\infty} \sum_{z_{2=1}}^{\infty} \cdots \sum_{z_{n=1}}^{\infty}\left\{\frac{(1-p)^{\sum_{i=1}^{n} z_{i} \Gamma(n+\alpha)}}{\left(\sum_{i=1}^{n} z_{i} x_{i}+\frac{1}{\bar{\beta}}\right)^{n+\alpha}}\right\}} \\
& =\frac{\sum_{j=1}^{\infty} \sum_{z_{1}=1}^{\infty} \sum_{z_{2}=1}^{\infty} \cdots \sum_{z_{n=1}}^{\infty}\left\{\frac{\frac{(1-p)^{j}}{j}(1-p)^{\sum_{i=1}^{\infty} z_{i}}}{\left(\sum_{i=1}^{n} z_{i} x_{i}+\frac{1}{\bar{\beta}}+j t\right)^{n+\alpha}}\right\}}{(-\ln p) \sum_{z_{1=1}}^{\infty} \sum_{z_{2}=1}^{\infty} \cdots \sum_{z_{n=1}}^{\infty}\left\{\frac{(1-p)^{\sum_{i=1}^{n} z_{i}}}{\left(\sum_{i=1}^{n} z_{i} x_{i}+\frac{1}{\bar{\beta}}\right)^{n+\alpha}}\right\}}
\end{aligned}
$$

\section{ESTIMATION UNDER LINEX LOSS FUNCTION}

Now we consider the estimation of parameter $\beta$ and survival function under the LINEX loss function. If we estimate parameter $\beta$ by, $\widehat{\beta}$ the corresponding LINEX loss function is defined as

$L(\hat{\beta})=\left[e^{c \Delta}-c \Delta-1\right], c \neq 0$

where $\Delta=\hat{\beta}-\beta$. For $c>0$, the loss function gives more weight age to overestimation where as for $c<0$, underestimation gets more weightage. When $c \rightarrow 0$, the LINEX loss function approaches to

$L(\hat{\beta})=\frac{c^{2}(\hat{\beta}-\beta)^{2}}{2}$

which is similar to the squared error loss function. 
Under the LINEX loss function the Bayes estimator of $\beta$ is given by

$\breve{\beta}_{L}=-\frac{1}{c} \ln E\left(e^{-c \beta}\right)$

where expectation is taken with respect to the posterior distribution of $\beta$. Now we have

$E\left(e^{-c \beta}\right)=\int_{0}^{\infty} e^{-c \beta} p^{*}(\beta \mid x) d \beta$

From equation (3.5) putting the value of $p^{*}(\beta \mid x)$ in equation (5.3) we get

$$
\begin{aligned}
& E\left(e^{-c \beta}\right) \\
& =\frac{\sum_{z_{1=1}}^{\infty} \sum_{z_{2=1}}^{\infty} \cdots \sum_{z_{n=1}}^{\infty}(1-p)^{\sum_{i=1}^{n} z_{i}} \beta^{n+\alpha-1} e^{-\beta\left(\sum_{i=1}^{n} z_{i} x_{i}+\frac{1}{\beta}+c\right)}}{\sum_{z_{1=1}}^{\infty} \sum_{z_{2=1}}^{\infty} \cdots \sum_{z_{n=1}}^{\infty}\left\{\frac{(1-p)^{\sum_{i=1}^{n} z_{i}} \Gamma(n+\alpha)}{\left(\sum_{i=1}^{n} z_{i} x_{i}+\frac{1}{\bar{\beta}}\right)^{n+\alpha}}\right\}}
\end{aligned}
$$

$$
=\frac{\sum_{z_{1=1}}^{\infty} \sum_{z_{2=1}}^{\infty} \cdots \sum_{z_{n=1}}^{\infty}\left\{\frac{(1-p)^{\sum_{i=1}^{n} z_{i}}}{\left(\sum_{i=1}^{n} z_{i} x_{i}+\frac{1}{\bar{\beta}}+c\right)^{n+\alpha}}\right\}}{\sum_{z_{1=1}}^{\infty} \sum_{z_{2=1}}^{\infty} \cdots \sum_{z_{n=1}}^{\infty}\left\{\frac{(1-p)^{\sum_{i=1}^{n} z_{i}}}{\left(\sum_{i=1}^{n} z_{i} x_{i}+\frac{1}{\bar{\beta}}\right)^{n+\alpha}}\right\}}
$$

Using the above expression (5.5) in (5.2) leads to the Bayes estimator of $\beta$.as

$\tilde{\beta}_{L}$

$$
=-\frac{1}{c} \ln \left\{\frac{\sum_{z_{1=1}}^{\infty} \sum_{z_{2=1}}^{\infty} \cdots \sum_{z_{n=1}}^{\infty}\left\{\frac{(1-p)^{\sum_{i=1}^{n} z_{i}}}{\left(\sum_{i=1}^{n} z_{i} x_{i}+\frac{1}{\bar{\beta}}+c\right)^{n+\alpha}}\right\}}{\sum_{z_{1}=1}^{\infty} \sum_{z_{2}=1}^{\infty} \cdots \sum_{z_{n=1}}^{\infty}\left\{\frac{(1-p)^{\sum_{i=1}^{n} z_{i}}}{\left(\sum_{i=1}^{n} z_{i} x_{i}+\frac{1}{\bar{\beta}}\right)^{n+\alpha}}\right\}}\right\}
$$

The Bayes risk of the estimator $\breve{\beta}_{L}$ under LINEX loss function is
$\rho\left(\tilde{\beta}_{L}\right)=c\left(\tilde{\beta}-\tilde{\beta}_{L}\right)$.

For obtaining the Bayes estimator of survival function $\mathrm{S}(\mathrm{t})$ under the LINEX loss function, we observe that

$$
\begin{aligned}
& e^{-c S(t)}=e^{\left[\ln \left[1-(1-p) e^{-\beta t}\right]\right]^{-\frac{c}{(\ln p)}}} \\
&=\left[1-(1-p) e^{-\beta t}\right]^{-\delta} \\
&=\sum_{j=0}^{\infty} \frac{\Gamma(\delta+j-1)}{\mathrm{j} ! \Gamma(\delta-1)}(1-p)^{j} e^{-j \beta t}
\end{aligned}
$$

where $\delta=\frac{c}{\ln p}$. Hence we observe that

$$
\begin{aligned}
& E\left[e^{-c S(t)}\right] \\
& =E\left[\sum_{j=0}^{\infty} \frac{\Gamma(\delta+j-1)}{\mathrm{j} ! \Gamma(\delta-1)}(1-p)^{j} e^{-j \beta t}\right] \\
& =\sum_{j=0}^{\infty} \frac{\Gamma(\delta+j-1)}{\mathrm{j} ! \Gamma(\delta-1)}(1-p)^{j} \sum_{z_{1}=1}^{\infty} \sum_{z_{2}=1}^{\infty} \cdots \sum_{z_{n=1}}^{\infty}(1-p)^{\sum_{i=1}^{n} z_{i}} \\
& \times \frac{\left.\int_{0}^{\infty} \beta^{n+\alpha-1} e^{-\beta\left(\sum_{i=1}^{n} z_{i} x_{i}+\frac{1}{\bar{\beta}}+j \beta t\right.}\right) d \beta}{\Gamma(n+\alpha) \sum_{z_{1=1}}^{\infty} \sum_{z_{2}=1}^{\infty} \cdots \sum_{z_{n=1}}^{\infty}\left\{\frac{(1-p)^{\sum_{i=1}^{n} z_{i}}}{\left(\sum_{i=1}^{n} z_{i} x_{i}+\frac{1}{\bar{\beta}}\right)^{n+\alpha}}\right\}} \\
& =\frac{(1-p)^{\sum_{i=1}^{n} z_{i}+j}}{\sum_{j=0}^{\infty} \frac{\Gamma(\delta+j-1)}{j ! \Gamma(\delta-1)} \sum_{z_{1=1}}^{\infty} \sum_{z_{2=1}}^{\infty} \cdots \sum_{z_{n=1}}^{\infty} \frac{\left(\sum_{i=1}^{n} z_{i} x_{i}+\frac{1}{\bar{\beta}}+j t\right.}{n+\alpha}} \\
& =\frac{(1-p)^{\sum_{i=1}^{n} z_{i}}}{\sum_{z_{1=1}}^{\infty} \sum_{z_{2=1}}^{\infty} \cdots \sum_{z_{n=1}}^{\infty}\left\{\frac{1}{\left(\sum_{i=1}^{n} z_{i} x_{i}+\frac{1}{\bar{\beta}}\right)^{n+\alpha}}\right\}}
\end{aligned}
$$

Hence the Bayes estimator of survival function under LINEX loss function is given by

$\tilde{S}_{L}(t)$

$$
=-\frac{1}{c} \ln \left[\frac{\sum_{j=0}^{\infty} \frac{\Gamma(\delta+j-1)}{j ! \Gamma(\delta-1)} \sum_{z_{1=1}}^{\infty} \sum_{z_{2=1}}^{\infty} \cdots \sum_{z_{n=1}}^{\infty} \frac{(1-p)^{\sum_{i=1}^{n} z_{i}+j}}{\left(\sum_{i=1}^{n} z_{i} x_{i}+\frac{1}{\bar{\beta}}+j t\right)^{n+\alpha}}}{\sum_{z_{1=1}}^{\infty} \sum_{z_{2=1}}^{\infty} \cdots \sum_{z_{n=1}}^{\infty}\left\{\frac{(1-p)^{\sum_{i=1}^{n} z_{i}}}{\left(\sum_{i=1}^{n} z_{i} x_{i}+\frac{1}{\bar{\beta}}\right)^{n+\alpha}}\right\}}\right]
$$

The risk associated with the Bayes estimator of survival function under LINEX loss function can be obtained as 
$\rho\left(\breve{\beta}_{L}\right)=c\left(\tilde{S}(t)-\tilde{S}_{L}(t)\right)$

\section{CONCLUding REMARK:}

CONCLUDING REMARKS The paper derives the posterior distribution and Bayes estimators assuming that the parameter $p$ is known. However, if $p$ is unknown, we may assume uniform or Beta prior distribution for $\mathrm{p}$ in interval $(0,1)$ and derive the conditional posterior distributions of $\mathrm{Q}$ given $\mathrm{p}$ and $\mathrm{p}$ given $\mathrm{u}$. Then the MCMC algorithm can be used to obtain the marginal distributions and Bayes estimators of parameters. The results of the paper can be easily extended when one considers type I censoring or type II censoring.

\section{REFERENCES:}

[1]. Adamidis, K., Loukas, S. (1998). A Lifetime Distribution with Decreasing Failure Rate. Statist. Probab. Lett. 39, 35-42.

[2]. Barlow, R.E., Marshall, A.W. (1964). Bounds for Distribution with Monotone Hazard Rate I and II. Ann. Math. Statist. 35, 1234-1274.

[3]. Barlow, R.E., Marshall, A.W. (1965). Tables of Bounds for Distribution with Monotone Hazard Rate. J. Amer. Statist. Assoc. 60, 872-890.

[4]. Barlow, R.E., Marshall, A.W. (1965). Tables of Bounds for Distribution with Monotone Hazard Rate. J. Amer. Statist. Assoc. 60, 872-890.

[5]. Barlow, R.E., Marshall, A.W., Proschan, F. (1963). Properties of Probability Distributions with Monotone Hazard Rate. Ann. Math. Statist. 34, 375-389.

[6]. Basu, A.P. and Ebrahimi, N. (1991). Bayesian Estimation Using Asymmetric Loss Function, Jour. Statist. Planning and Infer., 29, 21-31.

[7]. Chaturvedi, A. (1998). Robust Bayesian Analysis of the Exponential Failure Model, Pakistan Journal of Statistics, 14(2), 115-126.

[8]. Dahiya, R.C., Gurland, J. (1972). Goodness of Fit Tests for the Gamma and Exponential Distributions. Technometrics 14, 791801.

[9]. Dempster, A.P., Laird, N.M., Rubin, D.B., (1977). Maximum Likelihood from Incomplete Data Via the EM Algorithm (with discussion). J. Roy. Statist.

[10]. Gleser, L.J. (1989). The Gamma Distribution as a Mixture of Exponential Distributions. Amer. Statist. 43, 115-117.

[11]. Gurland, J., Sethuraman, J. (1994). Reversal of Increasing Failure Rates when Pooling Failure Data. Technometrics, 36, 416-418.

[12]. Johnson, N.L., Kotz, S., Kemp, A.W. (1993). Univariate Discrete Distributions. John Wiley. INC, New York.

[13]. Kuş, C. (2007). A New Lifetime Distribution. Computational Statistics \& Data Analysis, 51(9)

[14]. Lomax, K.S., 1954. Business Failure: Another Example of the Analysis of Failure Data. J. Amer. Statist. Assoc. 49, 847-852.

[15]. McLachlan, G.J., Krishnan, T., (2008). The EM Algorithm and Extensions, 2nd Edition. Wiley.
[16]. McNolty, F., Doyle, J., Hansen, E., 1980. Properties of the Mixed Exponential Failure Process. Technometrics 22, 555-565.

[17]. Nassar, M.M (1988). Two Properties of Mixtures of Exponential Distributions. IEEE Trans. Raliab. 37 (4), 383-385.

[18]. Pandey (B. N. 1997). Testimator of the Scale Parameter of the Exponential Distribution Using LINEX Loss Function. Communication in Statistics-Theory and Methods, 26(6), 21912202.

[19]. Proschan, F. (1963). Theoretical Explanation of Observed Decreasing Failure Rate. Technometrics 5, 375-383.

[20]. Saunders, S.C., Myhre, J.M. (1983). Maximum Likelihood Estimation for Two-Parameter Decreasing Hazard Rate Distributions Using Censored Data. J. Amer. Statist. Assoc. 78, 664-67.

[21]. Soliman, A. A. (2000). Comparison of Linex and Quadratic Bayes Estimators for the Rayleigh Distribution. Communication in Statistics-Theory and Methods, 29(1), 95-107.

[22]. Tahmasbi, R., Rezaei, S. (2008). A Two-Parameter Lifetime Distribution with Decreasing Failure Rate. Comput.Statist.Data Anal. 52, 3889-3901.

[23].Zellner, A. (1986). Bayesian Estimation and Prediction Using Asymmetric Loss Functions, Journal of American Statistical Association, 81, 446-451. 